\title{
Exciting Polaritons with Quantum Light
}

\author{
J. C. López Carreño, ${ }^{1}$ C. Sánchez Muñoz, ${ }^{1}$ D. Sanvitto, ${ }^{2}$ E. del Valle, ${ }^{1}$ and F. P. Laussy ${ }^{3,1, *}$ \\ ${ }^{1}$ Departamento de Física Teórica de la Materia Condensada and Condensed Matter Physics Center (IFIMAC), \\ Universidad Autónoma de Madrid, E-28049 Madrid, Spain \\ ${ }^{2}$ CNR NANOTEC-Institute of Nanotechnology, Via Monteroni, 73100 Lecce, Italy \\ ${ }^{3}$ Russian Quantum Center, Novaya 100, 143025 Skolkovo, Moscow Region, Russia
}

(Received 5 June 2015; published 4 November 2015)

\begin{abstract}
We discuss the excitation of polaritons-strongly coupled states of light and matter-by quantum light, instead of the usual laser or thermal excitation. As one illustration of the new horizons thus opened, we introduce "Mollow spectroscopy"- a theoretical concept for a spectroscopic technique that consists of scanning the output of resonance fluorescence onto an optical target-from which weak nonlinearities can be read with high precision even in strongly dissipative environments.
\end{abstract}

PACS numbers: 71.36.+c, 42.50.Ar, 42.50.Ct, 78.47.N-

Introduction.-One of the chief concerns of modern optics is to bring to fruition nontrivial quantum states of light $[1,2]$. This is typically achieved by driving a quantum system with a laser, turning light that is as classical as can be according to quantum mechanics into a nonclassical output $[3,4]$. In this Letter, we take the reversed stand of driving a weakly quantum system - or even a completely classical one-with a quantum source. Here, "classical" system means one that would yield classical states if excited by a laser, such as a harmonic oscillator with Hamiltonian $H_{a}=\hbar \omega_{a} a^{\dagger} a$. This simple case is still an important target as it describes, among other physical systems of interest, the single mode of a passive cavity or a field of noninteracting bosons such as plasmons [5]. To describe composite particles such as exciton polaritons [6], one then simply considers two harmonic oscillators $a$ and $b$ linearly coupled with strength $g$. Excitons being weakly interacting, with strength $U$, the polariton Hamiltonian becomes anharmonic [7]:

$$
H_{P}=\omega_{a} a^{\dagger} a+\omega_{b} b^{\dagger} b+g\left(a^{\dagger} b+a b^{\dagger}\right)+U b^{\dagger} b^{\dagger} b b .
$$

These systems are intrinsically open by nature, and driving them with a laser only allows for weak incursion, if any $[8,9]$, into the quantum regime. This is due to interactions being too small and dissipation too large for a laser to yield significant few-particle quantum effects. On the contrary, when driven by a quantum source, even a linear system is left in a strongly quantum state. This motivates us in introducing the paradigm of exciting polaritons with quantum light, cf. Fig. 1, opening a new chapter of the field already rich with mesoscopic quantum states, such as condensates [10], superfluids [11], Josephson oscillators [12,13], black hole analogues [14], polariton Higgs fields [15], etc. (see Ref. [16] for a review). Bringing such a versatile physics to the single-particle limit will allow the investigation of quantum simulators $[17,18]$ or logic with polariton Fock states $[19,20]$. We will now focus on two specific illustrations. Exciting a passive system, we realize a new brand of pure nonclassical steady states. Exciting interacting ones, we introduce a spectroscopic technique that extracts from photon statistics system parameters that are difficult to access through intensity or blueshift measurements.

Formalism.-With the ever increasing availability of quantum emitters, the question of their effect on a target is one of increasing theoretical interest. In the wake of the proposal for quantum optical spectroscopy [21], it has been shown that the statistics of light strongly affects the response of a system [22-26]. Related problems such as cloning [27] and the perfect excitation of a Fock state [28-30] are now topical. Our departure from the literature is to account self-consistently for the correlations imparted by the quantum source to the photons exciting the target. That is to say, instead of considering ad hoc quantum states of light, we include the exact dynamical properties of the light field as molded by its source. This allows us to include, for instance, correlations established through virtual processes, which is desirable, as they have the most interesting attributes [31]. The formalism to do that would then appear to simply include the source as a part of the system and solve for the dynamics of the joint exciting-excited components. An important requisite, however, is that the source is unaffected by the target: when an experimentalist shines light on a sample, the source's internal dynamics remains, in principle, unaffected by the presence, or not, of the sample. This is automatically realized in the conventional model for excitation by a laser since the latter is described by a $c$ number, which has no internal dynamics. For instance, exciting a two-level system $\sigma$ with a laser is simply modeled by the Hamiltonian [32]:

$$
H_{M}=\omega_{\sigma} \sigma^{\dagger} \sigma+\Omega \exp \left(i \omega_{L} t\right) \sigma+\Omega^{*} \exp \left(-i \omega_{L} t\right) \sigma^{\dagger},
$$

and regardless of the population, coherence, etc., effectively generated in the target, the attributes of the exciting laser (intensity $|\Omega|^{2}$ and frequency $\omega_{L}$ ) remain fixed as they are mere parameters of the model. In the fully quantized version [33], however, where the light field $\Omega e^{i \omega_{L} t}$ is 
upgraded to a Bose annihilation operator $a$, there is now a feedback from emitter $\sigma$ to the target $a$ [34]. This causes some dynamics of the supposed exciting laser that affects, in turn, the target, removing the asymmetry with the source that describes most experimental setups.

Concretely, our problem is the dynamic of the system described by Eq. (1) when it is excited by the output of the system described by Eq. (2). Separating the dynamics of coupled systems is tackled in the framework of cascaded systems [35], where the source output field is set as the target input field through equations of motion in the quantum Langevin form such that the source has no dependence on any operators from the target, which, on the contrary, depends on operators of the source. The derivation is detailed in the Supplemental Material [34] and yields a master equation $\partial_{t} \rho=i[\rho, H]+\sum_{i=1,2} \frac{\gamma_{i}}{2} \mathcal{L}_{c_{i}} \rho-\sqrt{\gamma_{1} \gamma_{2}}\left\{\left[c_{2}^{\dagger}, c_{1} \rho\right]+\right.$ $\left.\left[\rho c_{1}^{\dagger}, c_{2}\right]\right\}$ for the combined Hamiltonian $H=H_{P}+H_{M}$, with a Liouvillian in Lindblad form $\mathcal{L}_{c} \rho=\left(\gamma_{c} / 2\right)\left(2 c \rho c^{\dagger}-\right.$ $\left.\rho c^{\dagger} c-c^{\dagger} c \rho\right)$ and $c_{2}, c_{1}$, the operators from the source or target subsystems - in our case corresponding to $c_{1}=\sigma$ and $c_{2}=a$. We now tackle the aforementioned cases of interest.

Dissipative pure quantum state.-We first consider the simplest possible implementation: the excitation of a passive cavity (a harmonic oscillator) by the output of a weakly driven two-level system according to Eq. (2). Only two parameters rule this configuration: the ratio of decay rate of the target with the emission rate of the source, $\gamma_{a} / \gamma_{\sigma}$, and the pumping strength of the source $\Omega$ (also normalized to $\gamma_{\sigma}$ to keep the variables unitless). Figure 2 shows the states of the cavity field that can be reached, through (a) the photon statistics (color coded) and population (isolines) as well as (b) the purity of the state measured through $\operatorname{Tr}\left[\rho^{2}\right]$ (with zero corresponding to maximally mixed states and one to pure states). A large family of steadystate pure quantum states, i.e., with a wave function $|\psi\rangle_{\mathrm{ss}}=\sum_{i=0}^{\infty} \sqrt{\rho_{i, i}}|i\rangle$, can be obtained despite the driven-open nature of the system. Many of these states are nonclassical, sustaining quantum superpositions and sub-Poissonian fluctuations, depending on the interplay of quantum pumping and decay. The cut along the line I-II shows the transition from a regime where the target behaves according to its own classical nature (I) to one where it inherits instead the properties of the quantum source (II). In the former case, where the repetition rate of the emitter is larger than the decay rate, the many excitations that can be accumulated give predominance to the target, which grows a coherent state $\left(g^{(2)}=1\right)$ and exhibits the same PL spectrum as it would if it were excited classically. In the latter case, on the contrary, where the input is sparse, the target simply stores the excitation and reproduces it faithfully. This is the counterpart of the classically driven quantum dots in the Heitler regime that produce single photons with the coherence of the driving laser [36]. An interesting scenario lies in between, where the state fed in the cavity mixes characteristics of both its input and its recipient. Quantum states of the light field can thus be created with no need of quantum engineering, merely by exciting the system with resonance fluorescence, that is, the output of a driven two-level system. This realizes what one can refer to as a "dissipative Fock state $N$," defined as a steady state in an open environment qualitatively close to $\rho_{n m}=\delta_{N n} \delta_{N m}$. Together with the Fock states, we thus can find such steady states with a population larger than one and exhibiting antibunching $g^{(2)}<1$. Also, whenever $g^{(2)}<1 / 2$, the population is less than unity, thus still satisfying on average this flawed criterion for single particle excitation. Another cut, III-IV, on the isoline of average population unity, shows how antibunching increases as the amplitude of state $|1\rangle$ increases at the expense of vacuum and state $|3\rangle$, keeping the amplitude of state $|2\rangle$ the same, allowing for some quantum control in the steady state. Sources with an even higher quantum character [37] can yield steady states with $\rho_{22}>$ $\max \left(\rho_{11} / 2, \rho_{33}\right)$, that is, with fewer quantum fluctuations for two particles in the steady state than are normally allowed by spontaneous emission, thereby realizing the dissipative Fock state $N=2$. This is discussed further in the Supplemental Material [34].

Mollow spectroscopy.-We now consider another application of quantum excitation by driving the emitter in the Mollow regime of a spectral triplet [32] and in the presence of interactions $U$ for the target. The Mollow triplet is a treasure trove of photon correlations when selecting in

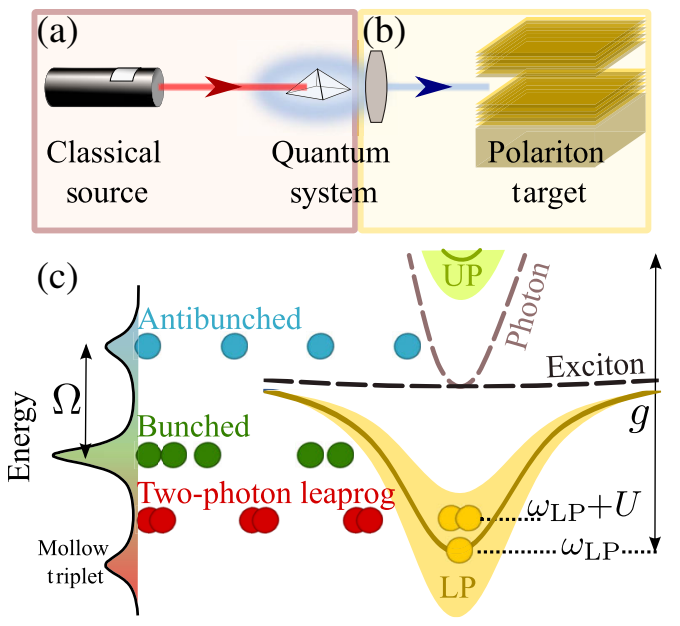

FIG. 1 (color online). Scheme of our proposal. (a) A typical optical excitation scenario of quantum optics, a laser excites a quantum system, e.g., a quantum dot. (b) Instead of the conventional scenario of also exciting polaritons with a laser, we excite polaritons with quantum light: specifically, from the output of the quantum system excited by the laser. (c) Mollow spectroscopy: the photoluminescence of a strongly driven two-level system provides the Mollow triplet, shown on the left with energy on the vertical axis. Various spectral windows provide different types of photon correlations, sketched here as photon balls with different temporal spacing. Exciting the lower polariton (LP) with leapfrog photon pairs allows us to measure accurately very small values of the interaction. 

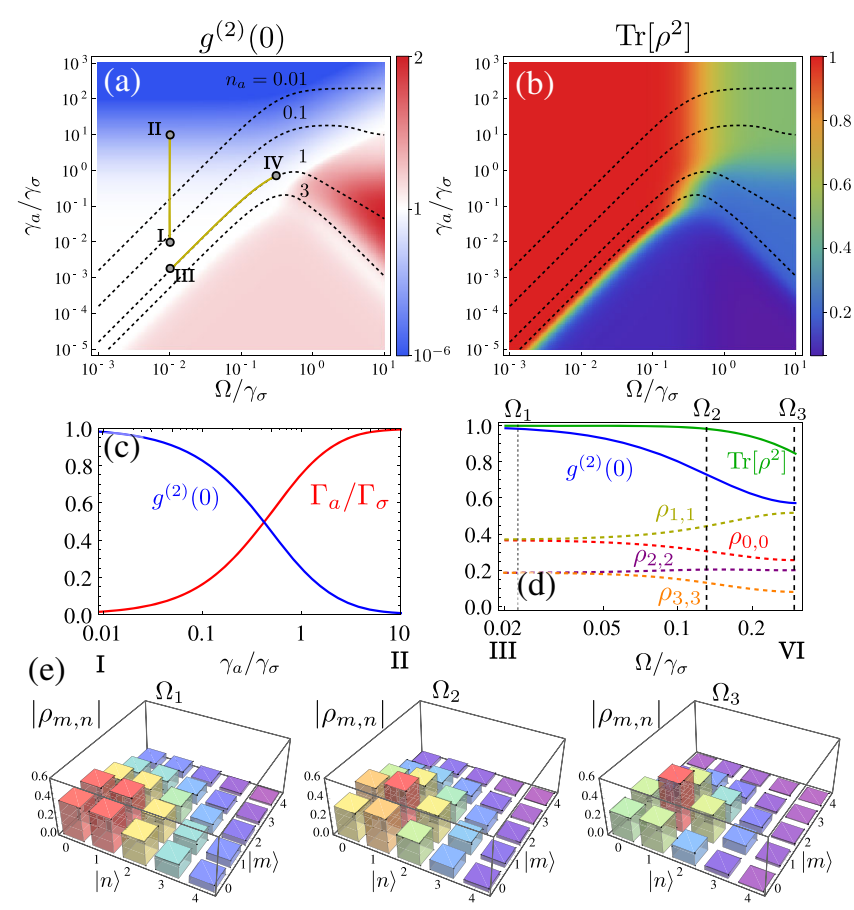

FIG. 2 (color online). Pure quantum states in the steady state. The red area of (b) shows where the system is in a pure state. The density plot of $g^{(2)}(0)$ in (a) shows that this corresponds to states with $g^{(2)} \leq 1$. Four isolines of constant populations are shown on both panels. (c) Antibunching and ratio of the effective linewidths of the target and source along the line I-II, showing a transfer of properties from the source to its target. (d) Antibunching, purity and diagonal elements $\rho_{n, n}=\langle n|\rho| n\rangle$ of the density matrix along the line III-IV. (e) Density matrices for three pumping powers. At $\Omega_{3}$, the system is in state $|1\rangle$, with over $60 \%$ probability, although not in a pure state anymore.

frequency windows [38]. Even though the emitter itself is a single-photon source, frequency selection allows us to access the full underlying dynamics that is otherwise averaged over to reduce to a mere antibunching. For instance, while photons from the side peaks are neatly antibunched, those from the central peak are slightly bunched; cf. Fig. 1(c). Less prone to attention, the emission halfway between the central peak and each satellite is, however, the most promising for applications. Photons at these frequencies originate from transitions between every other manifold by virtual processes, so-called leapfrog processes [38] that allow us to jump over the intermediate manifold in a direct two-photon deexcitation. This leads to superbunched strongly correlated pairs of identical photons, violating Cauchy-Schwarz and Bell's inequalities [31]. Photons in other frequency windows span intermediate cases. This has recently been spectacularly confirmed experimentally [39]. Such a rich variety of different quantum light from a single emitter can thus be scanned over the target to probe its response to all types of input, from single-photon light to superbunched, strongly correlated photon pairs. We call this new addition to the emerging field of photon-statistics spectroscopy [25] "Mollow spectroscopy."

The response of weakly interacting polaritons, Eq. (1), to classical light (a laser) shows that antibunching is extremely small and requires vanishing excitations [7], as stronger excitations lead to blueshift and bistability [40]. For this reason, unconventional polariton blockade mechanisms are favored [41,42], although these still require small pumping. In contrast, excitation with quantum light allows a strong response in statistics with small populations for all ranges of excitation and values of $U / \gamma_{a}$. Figure 3(a) shows the lower polariton population $n_{\mathrm{LP}}$ (the dot-dashed yellow line) and their second-order correlation function $g_{\mathrm{LP}}^{(2)}$ (the blue line) as the quantum source is scanned over the lower polariton (LP) branch, at energy $\omega_{\mathrm{LP}}=\left(\omega_{a}+\omega_{b}\right) / 2-$ $\sqrt{g^{2}-\left[\left(\gamma_{a}-\gamma_{b}\right) / 4\right]^{2}}$ (we take into account the small shift due to dissipation). Both the population and the statistics of the Mollow triplet are transferred to the polaritons, but with some deviations due to their interaction. These deviations are particularly marked in the polariton statistics, even down to very small values of $U / \gamma_{a}$, as seen in Fig. 3(b), where we plot the $L^{2}$ norm $\left\|g_{U=0}^{(2)}-g_{U \neq 0}^{(2)}\right\|_{2}$, i.e., integrating over frequencies the difference of photon statistics with and without nonlinearities. Variations of $\approx 1 \%$ are obtained for $U / \gamma_{a}=10^{-2}$. For lower values, this becomes a delicate measurement but still one within reach of state-of-the-art experiments [39]. The higher sensitivity of the nonlinearity in statistics than in population comes from the strongly correlated character of the leapfrog photon pairs, which optimize the effect of the interaction by consistently using the ideal number of photons (two) needed for it to manifest. The tunability of statistics from the Mollow triplet allows us, in particular, to extract the numerical value of the nonlinearity. This is achieved by measuring the change in photon statistics with the frequency of excitation. The maximum superbunching is provided by the two frequency windows between the central peak and the satellites. Photon pairs closer to the satellites lose this superbunching faster than those closer to the central peak. Comparing the response of the system when going in these two directions allows us to quantify small nonlinearities that are otherwise hidden in the radiative broadening. Thanks to the symmetry of the Mollow triplet, such a comparison can be conveniently implemented without the need for a calibration of the frequencies by using directly both sides of the triplet, rather than both sides of the superbunching peak. One can simply sweep the Mollow line shape onto the target and record its photon statistics. From these measurements, one then defines $f$ for the autoconvolution of the correlation function, $f=g^{(2)} * g^{(2)}$, i.e., $f\left(\omega_{0}\right)=\int_{-\infty}^{+\infty} g^{(2)}\left(\omega_{0}-\omega\right) g^{(2)}(\omega) d \omega$. The triangle inequality places the maximum of $f(\omega)$ at the value $\omega_{0}^{*}$ that minimizes the asymmetry of $g^{(2)}(\omega)$ around $\omega_{0}-\omega_{\mathrm{LP}}$. When the leapfrog processes are sufficiently well defined (see below), we find that the shift is precisely given by the two-polariton interaction: 

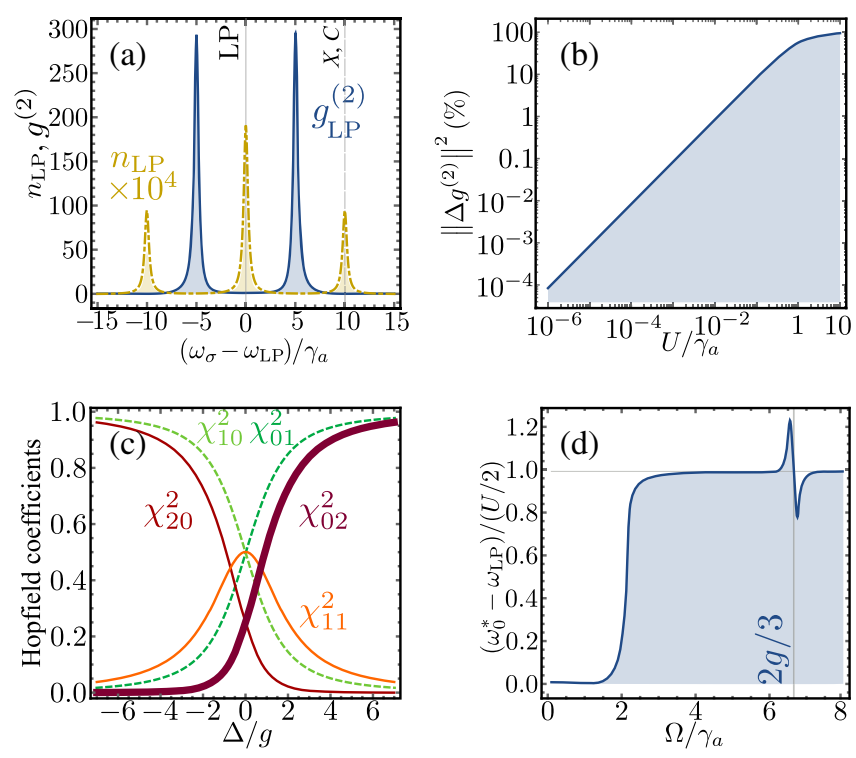

FIG. 3 (color online). Mollow spectroscopy of weakly interacting polaritons. (a) Population (dot-dashed yellow line) and photon-statistics (solid blue line) when scanning the Mollow triplet onto the lower polariton branch. (b) Magnitude of the deviation from the noninteracting polariton statistics as a function of $U / \gamma_{a}$. (c) Hopfield coefficients $\chi_{n m}$ of relative light (n)-matter ( $m$ ) content, as a function of detuning. (d) Mollow splitting required for the measurement of $\omega_{0}$ to be $\Omega$ independent, allowing an absolute measurement of $U$.

$$
\omega_{0}^{*}=\omega_{\mathrm{LP}}+2 U \chi_{02}^{2},
$$

where $\chi_{02}$ is the two-polariton Hopfield coefficient for the state $|02\rangle$ of two excitons and zero photons. The closed-form expression of $\chi_{02}$ in terms of detuning is too cumbersome to be given here, but the value is straightforwardly obtained by diagonalization of Eq. (1) and is in excellent approximation given by $\chi_{01}^{2}$; cf. Fig. 3(c). In the case of an anharmonic oscillator with no exciton-photon structure, the shift would be directly given by $2 U$. There is thus no dependence of the measurement $\omega_{0}^{*}$ on the population or other dynamical variables and it is therefore absolute, unlike the blueshift from a classical driving that requires knowledge of the effective laser intensity. This is not the only advantage that powers Mollow spectroscopy. A strong pumping of polaritons leads to several complications that hinder full or compelling information, such as heating, phase-space filling [43], loss of strong coupling [44], population of an exciton reservoir contributing the bulk of the blueshift $[45,46]$, exciton-photon fraction deviating from the Hopfield coefficients [47], etc. Since polariton interactions provide the foundation for nonlinear effects that constitute much of the polariton literature, the question of their nature and magnitude could be regarded as one of the most important open problems of the field [48-50]. In contrast, the excitation of the same system with the Mollow triplet is clean of all such complications of high densities. Since it recourses to the minimum amount of polaritons required to poke the interaction (two), Mollow spectroscopy acts as a "probe" in the ultimate sense of the term, with as little disturbance as possible.

For Eq. (3) to hold, the Mollow triplet must be such that leapfrog processes are not mixed with other types of less correlated emission, leading to some departure from a shift ruled wholly by the two-polariton interaction. Figure 3(d) shows the splitting required for Eq. (3) to be accurate, namely, in the plateau when $\Omega \geq 3 \gamma_{a}$. Even at low splitting, from a power dependence, one can estimate the nonlinearity. For large Mollow splittings against the Rabi splitting, there is a resonance in $g^{(2)}$ at $\Omega / \gamma_{a}=3 g / 2$ when the lower leapfrog excites the lower polariton branch, while the upper Mollow satellite excites the upper polariton branch.

Conclusions and Perspectives.-We have shown how exciting a system with quantum light opens new perspectives in several areas of quantum physics. The nature of the quantum excitation here is such that dynamical correlations from a source are self-consistently mapped to the target, rather than assuming initial conditions set by hand and, for this reason, bound to particular cases. Specifically, our approach describes realistic quantum light in its full complexity, rather than limiting it to particular and often much simpler cases (such as Fock or squeezed states). This takes advantage in particular of the dynamical aspects of the source, such as virtual processes of the emitter. It also brings several improvements in the theoretical description of an increasingly pivotal problem, the use of quantum sources in practical applications and their interfacing with other systems. As illustrations of the benefits of exciting with quantum light, we have shown how to realize pure nonclassical steady states by driving a cavity with a singlephoton source, and we have introduced a new type of spectroscopy by the suppression of fluctuations. These results suggest numerous other applications, including the spin, performing quantum pump-probe and two-tone spectroscopy with delay between quantum correlated beams, probing and exciting continuous fields or their resonant configurations such as optical parametric oscillators, and driving other quantum systems, e.g., two-level systems, coupled cavities, polariton circuits, etc., each of them amenable to several types of quantum light. The emitter of Fock states recently proposed by some of the authors [37], releasing all its energy in bundles of $N$ photons, should be a key resource for quantum excitation. Beyond the exotic states of light already alluded to, this may open the door to new classes of excitations, such as the correlated electron-hole clusters, the so-called dropletons [51], discovered within the limitations of classical excitations by theoretical deduction.

We thank Amir Rahmani and Kai Müller for the discussions and acknowledge funding by the Spanish MINECO (FPI and RyC) and the EU with the ERC POLAFLOW Project No. 308136. 
*fabrice.laussy@gmail.com

[1] J. L. O'Brien, A. Furusawa, and J. Vuckovic, Nat. Photonics 3, 687 (2009).

[2] M. Bertolotti, F. Bovino, and C. Sibilia, Prog. Opt. 60, 1 (2015).

[3] V. V. Dodonov, J. Opt. B 4, R1 (2002).

[4] K. Müller, A. Rundquist, K. A. Fischer, T. Sarmiento, K. G. Lagoudakis, Y. A. Kelaita, C. Sánchez Muñoz, E. del Valle, F. P. Laussy, and J. Vuckovic, Phys. Rev. Lett. 114, 233601 (2015).

[5] F. Javier García de Abajo, Nature (London) 483, 417 (2012).

[6] C. Weisbuch, M. Nishioka, A. Ishikawa, and Y. Arakawa, Phys. Rev. Lett. 69, 3314 (1992).

[7] A. Verger, C. Ciuti, and I. Carusotto, Phys. Rev. B 73, 193306 (2006).

[8] V. Ardizzone, M. Abbarchi, A. Lemaitre, I. Sagnes, P. Senellart, J. Bloch, C. Delalande, J. Tignon, and P. Roussignol, Phys. Rev. B 86, 041301(R) (2012).

[9] T. Boulier, M. Bamba, A. Amo, C. Adrados, A. Lemaitre, E. Galopin, I. Sagnes, J. Bloch, C. Ciuti, E. Giacobino et al., Nat. Commun. 5, 3260 (2014).

[10] J. Kasprzak, M. Richard, S. Kundermann, A. Baas, P. Jeambrun, J. M. J. Keeling, F. M. Marchetti, M. H. Szymanska, R. André, J. L. Staehli et al., Nature (London) 443, 409 (2006).

[11] A. Amo, J. Lefrère, S. Pigeon, C. Adrados, C. Ciuti, I. Carusotto, R. Houdré, E. Giacobino, and A. Bramati, Nat. Phys. 5, 805 (2009).

[12] K. G. Lagoudakis, B. Pietka, M. Wouters, R. André, and B. Deveaud-Plédran, Phys. Rev. Lett. 105, 120403 (2010).

[13] M. Abbarchi, A. Amo, V. G. Sala, D. D. Solnyshkov, H. Flayac, L. Ferrier, I. Sagnes, E. Galopin, A. Lemaître, G. Malpuech et al., Nat. Phys. 9, 275 (2013).

[14] H. S. Nguyen, D. Gerace, I. Carusotto, D. Sanvitto, E. Galopin, A. Lemaître, I. Sagnes, J. Bloch, and A. Amo, Phys. Rev. Lett. 114, 036402 (2015).

[15] E. S. Sedov, I. V. Iorsh, S. M. Arakelian, A. P. Alodjants, and A. Kavokin, Phys. Rev. Lett. 114, 237402 (2015).

[16] I. Carusotto and C. Ciuti, Rev. Mod. Phys. 85, 299 (2013).

[17] T. Byrnes, K. Yan, and Y. Yamamoto, New J. Phys. 13, 113025 (2011).

[18] T. Byrnes, S. Koyama, K. Yan, and Y. Yamamoto, Sci. Rep. 3, 2531 (2013).

[19] D. Ballarini, M. D. Giorgi, E. Cancellieri, R. Houdré, E. Giacobino, R. Cingolani, A. Bramati, G. Gigli, and D. Sanvitto, Nat. Commun. 4, 1778 (2013).

[20] C. Antón, T. C. H. Liew, J. Cuadra, M. D. Martín, P. S. Eldridge, Z. Hatzopoulos, G. Stavrinidis, P. G. Savvidis, and L. Viña, Phys. Rev. B 88, 245307 (2013).

[21] M. Kira and S. W. Koch, Phys. Rev. A 73, 013813 (2006).

[22] A. Carmele, A. Knorr, and M. Richter, Phys. Rev. B 79, 035316 (2009).

[23] M. Kira, S. W. Koch, R. P. Smith, A. E. Hunter, and S. T. Cundiff, Nat. Phys. 7, 799 (2011).

[24] M. Aßmann and M. Bayer, Phys. Rev. A 84, 053806 (2011).

[25] T. Kazimierczuk, J. Schmutzler, M. Aßmann, C. Schneider, M. Kamp, S. Höfling, and M. Bayer, Phys. Rev. Lett. 115, 027401 (2015).

[26] S. Mukamel and K. E. Dorfman, Phys. Rev. A 91, 053844 (2015).
[27] A. P. Alodjants and S. M. Arakelian, Int. J. Mod. Phys. B 20, 1593 (2006).

[28] R. Johne and A. Fiore, Phys. Rev. A 84, 053850 (2011).

[29] B. Srivathsan, G. K. Gulati, A. Ceré, B. Chng, and C. Kurtsiefer, Phys. Rev. Lett. 113, 163601 (2014).

[30] C. Liu, Y. Sun, L. Zhao, S. Zhang, M. M. T. Loy, and S. Du, Phys. Rev. Lett. 113, 133601 (2014).

[31] C. Sánchez Muñoz, E. del Valle, C. Tejedor, and F. P. Laussy, Phys. Rev. A 90, 052111 (2014).

[32] B. R. Mollow, Phys. Rev. 188, 1969 (1969).

[33] E. del Valle and F. P. Laussy, Phys. Rev. Lett. 105, 233601 (2010).

[34] See Supplemental Material at http://link.aps.org/ supplemental/10.1103/PhysRevLett.115.196402 for the formalism, exciting with a $N$-photon emitter, going beyond the single-mode approximation and a discussion of the parameters.

[35] G. W. Gardiner and P. Zoller, Quantum Noise, 2nd ed. (Springer-Verlag, Berlin, 2000).

[36] C. Matthiesen, M. Geller, C. H. H. Schulte, C. L. Gall, J. Hansom, Z. Li, M. Hugues, E. Clarke, and M. Atatüre, Nat. Commun. 4, 1600 (2013).

[37] C. Sánchez Muñoz, E. del Valle, A. G. Tudela, K. Müller, S. Lichtmannecker, M. Kaniber, C. Tejedor, J. Finley, and F. Laussy, Nat. Photonics 8, 550 (2014).

[38] A. Gonzalez-Tudela, F. P. Laussy, C. Tejedor, M. J. Hartmann, and E. del Valle, New J. Phys. 15, 033036 (2013).

[39] M. Peiris, B. Petrak, K. Konthasinghe, Y. Yu, Z. C. Niu, and A. Muller, Phys. Rev. B 91, 195125 (2015).

[40] G. Messin, J. P. Karr, A. Baas, G. Khitrova, R. Houdré, R. P. Stanley, U. Oesterle, and E. Giacobino, Phys. Rev. Lett. 87, 127403 (2001).

[41] T. C. H. Liew and V. Savona, Phys. Rev. Lett. 104, 183601 (2010).

[42] M. Bamba, A. Imamoğlu, I. Carusotto, and C. Ciuti, Phys. Rev. A 83, 021802(R) (2011).

[43] S. Schmitt-Rink, D. S. Chemla, and D. A. B. Miller, Phys. Rev. B 32, 6601 (1985).

[44] R. Houdré, J. L. Gibernon, P. Pellandini, R. P. Stanley, U. Oesterle, C. Weisbuch, J. O'Gorman, B. Roycroft, and M. Ilegems, Phys. Rev. B 52, 7810 (1995).

[45] L. Ferrier, E. Wertz, R. Johne, D. D. Solnyshkov, P. Senellart, I. Sagnes, A. Lemaître, G. Malpuech, and J. Bloch, Phys. Rev. Lett. 106, 126401 (2011).

[46] G. Christmann, G. Tosi, N. G. Berloff, P. Tsotsis, P. S. Eldridge, Z. Hatzopoulos, P. G. Savvidis, and J. J. Baumberg, Phys. Rev. B 85, 235303 (2012).

[47] Y. V. Kartashov, V. V. Konotop, and L. Torner, Phys. Rev. B 86, 205313 (2012).

[48] G. Rossbach, J. Levrat, E. Feltin, J.-F. Carlin, R. Butté, and N. Grandjean, Phys. Rev. B 88, 165312 (2013).

[49] P. Cilibrizzi, H. Ohadi, T. Ostatnicky, A. Askitopoulos, W. Langbein, and P. Lagoudakis, Phys. Rev. Lett. 113, 103901 (2014).

[50] Y. Sun, Y. Yoon, M. Steger, G. Liu, L. N. Pfeiffer, K. West, D. W. Snoke, and K. A. Nelson, arXiv:1508.06698.

[51] A. E. Almand-Hunter, H. Li, S. T. Cundiff, M. Mootz, M. Kira, and S. W. Koch, Nature (London) 506, 471 (2014). 